\title{
Patterning pallet arrays for cell selection based on high- resolution measurements of fluorescent biosensors
}

\author{
Hamed Shadpoura , Jon S. Zawistowski ${ }^{\mathrm{b}}$, Annadele Herman ${ }^{\mathrm{a}}$, Klaus Hahn ${ }^{\mathrm{b}}$, and Nancy L. \\ Allbritton ${ }^{a, b, c,}$, \\ aDepartment of Chemistry, University of North Carolina, Chapel Hill, North Carolina 27599, USA \\ bDepartment of Pharmacology, University of North Carolina, Chapel Hill, North Carolina 27599, \\ USA
}

'Department of Biomedical Engineering, University of North Carolina, Chapel Hill, 27599, North Carolina, USA, and North Carolina State University, Raleigh, North Carolina 27695, USA

\begin{abstract}
Pallet arrays enable cells to be separated while they remain adherent to a surface and provide a much greater range of cell selection criteria relative to that of current technologies. However there remains a need to further broaden cell selection criteria to include dynamic intracellular signaling events. To demonstrate the feasibility of measuring cellular protein behavior on the arrays using high resolution microscopy, the surfaces of individual pallets were modified to minimize the impact of scattered light at the pallet edges. The surfaces of the three-dimensional pallets on an array were patterned with a coating such as fibronectin using a customized stamping tool. Micropatterns of varying shape and size were printed in designated regions on the pallets in single or multiple steps to demonstrate the reliability and precision of patterning molecules on the pallet surface. Use of a fibronectin matrix stamped at the center of each pallet permitted the localization of H1299 and mouse embryonic fibroblast (MEF) cells to the pallet centers and away from the edges. Compared to pallet arrays with fibronection coating the entire top surface, arrays with a central fibronectin pattern increased the percentage of cells localized to the pallet center by 3-4 fold. Localization of cells to the pallet center also enabled the physical separation of cells from optical artifacts created by the rough pallet side walls. To demonstrate the measurement of dynamic intracellular signaling on the arrays, fluorescence measurements of high spatial resolution were performed using a RhoA GTPase biosensor. This biosensor utilized fluorescence resonance energy transfer (FRET) between cyan fluorescent protein (CFP) and yellow fluorescent protein (YFP) to measure localized RhoA activity in cellular ruffles at the cell periphery. These results demonstrated the ability to perform spatially resolved measurements of fluorescence-based sensors on the pallet arrays. Thus, the patterned pallet arrays should enable novel cell separations in which cell selection is based on complex cellular signaling properties.
\end{abstract}

(C) 2011 Elsevier B.V. All rights reserved.

“Corresponding author nlallbri@ unc.edu. Fax: 919-962-2388, Phone: 919-966-2291.

Publisher's Disclaimer: This is a PDF file of an unedited manuscript that has been accepted for publication. As a service to our customers we are providing this early version of the manuscript. The manuscript will undergo copyediting, typesetting, and review of the resulting proof before it is published in its final citable form. Please note that during the production process errors may be discovered which could affect the content, and all legal disclaimers that apply to the journal pertain.

Appendix A. Supplementary data

Supplementary data associated with this article can be found in the online version at doi:xxxxxxxxxxxxxxxxxxxx. 


\section{Keywords}

pallet array; cell separation; patterned surfaces; FRET; cell sorting; micro-contact printing

\section{Introduction}

There is currently great interest in studying the mechanisms of spatially isolated behaviors in cells such as calcium sparks and waves, pseudopodia extension and retraction, invadopodia dynamics, focal adhesion formation, endoplasmic reticulum assembly and disassembly among many others. A plethora of probes have now been developed to track this localized signaling behavior for example fluorescent monitors for calcium and other ions, FRETbased indicators for kinase proteases and GTPases, translocation probes to monitor protein domain movement, fluorescent highlighters to identify protein complex age and assembly/ disassembly, and small metabolites sensors [1-3]. Biosensor design and synthesis has been particularly successful for kinases [4] which add a phosphoryl to a protein or lipid and GTPases such as the Rho family [5] which cycle between active and inactive states in a nucleotide-dependent manner. Live cell imaging studies have revealed the subcellular spatial and temporal dynamics of activation for these enzymes in single cells. Use of the biosensors has also revealed the diversity of signaling among single cells within a population. While these measurements have been performed at the single-cell level, cells identified as having interesting signaling variants have not been obtainable as a pure population and are thus unavailable for future studies. This is largely due to the spatially and temporally dynamic behavior of cellular signaling enzymes and the absence of cell separation technologies compatible with cell selection based on high resolution measurements in both time and space. Methods such as fluorescence-activated cell sorting (FACS) or magnetic-activated cell sorting (MACS) offer no or extremely limited spatial and temporal measurements of biosensor behavior in cells. However, much utility lies in the use of live cell biosensors for the screening and isolation of cells displaying unique signaling behaviors in response to altered environmental conditions, drug applications, or other perturbations.

Pertz and colleagues have previously reported an intra-molecular fluorescence resonance energy transfer (FRET) biosensor for the small GTPase Rho [6]. Upon RhoA activation by GTP-loading, RhoA interacts with a tethered Rho-binding domain (RBD), altering the relative orientation of cyan fluorescent protein (CFP) and yellow fluorescent protein (YFP) within the tether. In migrating mouse embryonic fibroblasts (MEFs), RhoA activation is present in a tight band of activity at the leading edge of the cell as well as transiently during tail retraction [6]. With this biosensor and others, the spatial and temporal relationship between RhoA activity and the GTPases Rac1 and Cdc42 was elucidated, showing that RhoA is activated first among these GTPases at the cell edge, followed by Rac1 and Cdc42 activation occurring at a zone away from the edge with a $\sim 40$ second time delay [7]. Genetic or pharmacological perturbation of these spatial or temporal activation signatures followed by selection and separation of cells with unique molecular activation states would be of high utility in investigations of RhoA signaling.

Microarrays varied in type, size, and function have been a part of the biomedical experimentalists' tool box for many years [8,9]. Arrays of releasable elements or pallets which are made of photoresists such as SU8 and 1002F were recently introduced for the separation of adherent cells [10-13]. Cells arrayed on the releasable elements can be screened by conventional microscopy-based assays and instrumentation. Selected cells can then be released from the underlying substrate, collected and expanded [9]. Despite the advantages that these releasable microarrays provide in screening and separating cells [8-13], the technology possesses limited utility for separations based on fluorescence 
measurements of high spatial resolution, for example, the measurement of subcellular signaling events. This constraint is primarily due to intense light scattering at the pallet edges which interferes with fluorescence measurements in adjacent cellular regions as well as to the very short working distance of the required high-numerical aperture (NA), oilbased objectives. The origin of the light scatter is the differing refractive indices between the pallet and surrounding medium. Light scattering from the pallet edges into the microscope objective during imaging is likely enhanced by the rough pallet side walls. Further, the greater roughness at the junction of the pallet surface and side wall results in a preference for cell attachment near the edges of the pallet where scattering is at its greatest intensity. The small working distance of the high NA objective also necessitates an array of minimum thickness. Thus the acquisition of high resolution fluorescence images of cells on the pallets to screen and select cells based on biosensor readouts of subcellular processes has not been possible.

The goal of this work was to demonstrate the feasibility of high resolution imaging of RhoA activity in cells on the pallet arrays. To achieve this goal and minimize optical artifacts, the pallet surfaces were patterned to direct cell attachment to the center of the pallet and spatially separate cellular fluorescence from the scattered light. The novelty of this work lies in the successful adaptation of the arrayed releasable elements or pallets so that the elements were compatible with high resolution imaging of fluorescent biosensors over time. This includes the adaptation of the array for the high NA objectives as well as surface patterning to spatially segregate light scatter. These modifications enable highly sensitive, temporally, and spatially resolved fluorescence measurements of cells cultured on the pallet arrays which has the potential to enable cell selection and separation based on complex, dynamic protein behavior, a feat not currently possible. This achievement would greatly broaden the type of selection criteria used to separate a mixed population of cells potentially identifying cells with variant pathways or unique drug responses.

\section{Experimental}

\subsection{Materials}

Dulbecco's Modified Eagle Medium (DMEM), fetal bovine serum (FBS), L-glutamine, penicillin/streptomycin, puromycin, doxycycline, Ham's F-12K medium without phenol red, phosphate buffered saline (PBS) and 0.05\% trypsin with EDTA were obtained from Invitrogen (Carlsbad, CA). HEPES buffer was purchased from Mediatech, Inc. (Manassas, VA). EPON resin 1002F (phenol, 4,4'-(1-methylethylidene)bis-, polymer with 2,2'-[(1methylethylidene) bis(4,1-phenyleneoxymethylene]bis[oxirane]) was acquired from MillerStephenson (Sylmar, CA). SU8 photoresist and SU8 developer (1-methoxy-2-propyl acetate, also used for 1002F) were from MicroChem Corp. (Newton, MA). Heptadecafluoro-1,1,2,2tetrahydrodecyl trichlorosilane was from Gelest Inc. (Morrisville, PA). Human plasma fibronectin was purchased from Millipore Corporation (Billerica, MA). Unless otherwise said in the text, all other chemicals were obtained from Fisher Scientific (Pittsburgh, PA).

\subsection{Fabrication of pallet arrays}

Arrays of $1002 \mathrm{~F}$ (130 $\mu \mathrm{m}$ sides, $50 \mu \mathrm{m}$ spacing, and $50 \mu \mathrm{m}$ height unless stated otherwise) were fabricated on glass cover slips (Warner Instruments, Hamden, CT) as described previously [14]. The use of number one cover slips (thickness $\sim 150 \mu \mathrm{m}$ ) was necessary in order to perform cell fluorescence measurements with high numerical aperture objectives. Chambers ( $25 \mathrm{~mm}$ size) surrounding the arrays were constructed from poly(dimethyl siloxane) (PDMS) using a Sylgard 184 silicone elastomer kit (Dow Corning, Midland, MI) as described previously [10]. To eliminate cell attachment between the pallets, virtual air walls were created between the pallets using a vapor-phase deposition with 
heptadecafluoro-1,1,2,2-tetrahydrodecyl trichlorosilane overnight as described previously [8]. After an overnight coating, the arrays were kept on a $120^{\circ} \mathrm{C}$ hot plate for 2 hours and the remaining chemical residue washed with ethanol and the array dried with a $\mathrm{N}_{2}$ stream. To enhance adhesion of matrices to the pallet, the pallet surfaces were roughened for $30 \mathrm{~s}$ as described previously [13]. The arrays were then washed with water and ethanol and dried in stream of $\mathrm{N}_{2}$. The arrays were stored in a vacuum desiccator until use.

\subsection{Fabrication of PDMS stamps}

1002F molds in various shapes and sizes (see Results and Discussion) for forming the PDMS stamps were fabricated and coated overnight by vapor-phase deposition with heptadecafluoro-1,1,2,2-tetrahydrodecyl trichlorosilane as described above. A 1:8 (catalyst:monomer) PDMS mixture was layered over the 1002F mold. In order to prevent shrinking or expansion of the PDMS stamp after release from the mold, the uncured PDMS mixture was covered with a $50-\mu \mathrm{m}$ thick $1002 \mathrm{~F}$ film fabricated on $1-\mathrm{mm}$ thick microscope glass slide. The assembly was then degassed for $1 \mathrm{~h}$ and cured for one hour at $65^{\circ} \mathrm{C}$. After slowly cooling to room temperature, the PDMS stamp was carefully cut and peeled from the $1002 \mathrm{~F}$ mold while it remained bonded to the underlying support of $1002 \mathrm{~F}$ film on glass. The PDMS stamp was roughened for $25 \mathrm{~s}$ as described previously [13]. After gently washing with water and ethanol followed by drying under an $\mathrm{N}_{2}$ stream, the PDMS stamps were placed in an air-plasma cleaner (Harrick PDC-001, Ithaca, New York) for $20 \mathrm{~min}$ immediately before use.

\subsection{Alignment of the PDMS stamp and pallet array}

A customized alignment tool was constructed to mate with the differential interference contrast (DIC) assembly of a microscope (Nikon TE 2000). The tool was used to precisely align the underlying pallet array with the PDMS stamp (Figs. 1A \& S1). The device was comprised of a Teflon tube attached to a transparent polystyrene dish. During stamping, one end of this Teflon tube was temporarily mounted to the DIC assembly on a microscope stage. On the other side of the polystyrene lid, a Teflon ring with a soft silicon O-ring cover was assembled for attachment to the PDMS stamp. The PDMS stamp with its supporting $1002 \mathrm{~F} / \mathrm{glass}$ base was held in place by application of a vacuum. The stamp could be moved along the $z$-axis to load ink and for stamping. The pallet array on the microscope stage could be moved in the $x-y$ directions alignment to the stamp.

Fibronectin ( $500 \mu \mathrm{g} / \mathrm{mL}$ in PBS, $0.5 \mathrm{~mL}$ ) was spread on a lint-free wipe (TechniCloth, ITW Texwipe, Mahwah, NJ) inside a culture dish and used as a stamping pad. Inking of the PDMS stamp with fibronectin was performed by placing the PDMS stamp and stamping pad in contact for approximately $10 \mathrm{~s}$ on the microscope stage using the alignment tool described above. Alignment of the stamp to a pallet array on the microscope was then performed under low magnification (e.g., 20x). After obtaining an acceptable alignment between the stamp and pallet array, the inked stamp was depressed onto an array for $30 \mathrm{~s}$. The stamped arrays were dried in a tissue culture hood for at least 30 minutes and then sterilized by exposure to UV light for 20 minutes in a standard tissue culture hood.

\subsection{Cell Culture}

eGFP-expressing H1299 (small cell lung carcinoma) and HeLa cells (ATCC, Manassas, VA) were cultured in DMEM supplemented with $10 \%$ FBS, $584 \mathrm{mg} / \mathrm{L}$ L-glutamine, 100 units $/ \mathrm{mL}$ penicillin and $100 \mu \mathrm{g} / \mathrm{mL}$ streptomycin at $37^{\circ} \mathrm{C}$ in a humidified $5 \% \mathrm{CO}_{2}$ atmosphere.

To create an inducible RhoA biosensor cell line, Tet-Off MEFs (Clontech-Takara Biosciences \#C3018-1) were transduced with retrovirus produced with the pBabe-Purotet- 
CMV vector backbone[15] in ecotropic LinXE packaging cells. A $10 \mu \mathrm{g} / \mathrm{ml}$ puromycin treatment was used to select for stable incorporation of the biosensor. RhoA biosensorexpressing MEFs were maintained in DMEM with 10\% FBS. $1 \mu \mathrm{g} / \mathrm{ml}$ doxycycline was used to repress the expression of the RhoA biosensor during normal cell culture passages. For induction of biosensor expression, cells were centrifuged to remove doxycycline and replated at $10^{4}$ cells per $10 \mathrm{~cm}$ dish 48 hours prior to imaging. 24 hours prior to imaging, $10^{4}$ RhoA biosensor-expressing MEFs were trypsinized and plated on pallets stamped with fibronectin. Imaging was performed in Ham's F-12K medium without phenol red but supplemented with $2 \%$ FBS and $10 \mathrm{mM}$ HEPES.

\subsection{Brightfield and fluorescence microscopy}

For experiments on cells without a GFP or expressing only eGFP, bright field and DIC micrographs of cells incubated on the 1002F pallet arrays were captured using an inverted microscope (Nikon TE 2000) with a cooled CCD camera (Coolsnap HQ², Photometrics, Tucson, AZ) and a standard fluorescein filter set. All images were collected using Elements software (Nikon).

RhoA activation levels were measured following CFP excitation by monitoring the ratio of the fluorescence emission of YFP to CFP fluorescence intensity. Biosensor imaging was performed on an Olympus IX81 inverted epifluorescence microscope using an oilimmersion, Olympus 40x UPlan FLN 1.3 NA DIC lens, Coolsnap HQ ${ }^{2}$ CCD camera (Photometrics) and Metamorph software (Molecular Devices, Downingtown, PA). Cells were illuminated using a $100 \mathrm{~W}$ mercury arc lamp through an ND 1.5 neutral density filter. The following filter sets were used (Chroma, Bellows Falls, VT): CFP: D436/20, D470/40; YFP: D436/20, HQ535/30. A custom dichroic mirror (“Quad-Custom” Lot\# 511112038) manufactured by Chroma was utilized with these filter sets. Two images were acquired for each time point with the following exposure times: CFP (1.0 s) and YFP (0.5 s). Image sets were taken at $30 \mathrm{~s}$ intervals. Images were obtained using $2 \times 2$ binning on a 1,392 $\times 1,040$ pixel array at $40 \mathrm{X}$ magnification, providing a spatial resolution of $0.33 \mu \mathrm{m}$. Image registration, ratio calculations and photobleach correction were performed as described previously [16].

\section{Results and discussion}

\subsection{Patterning the surfaces of arrayed pallets with inks}

A customized alignment tool for precise stamping of biomolecules on micron-size structures was fabricated (Fig. 1A). The tool was mounted on the stage of a microscope to permit alignment and controlled lowering of an inked stamp onto an underlying pallet array. Figs. 1B \& C demonstrate two examples of the PDMS stamps aligned over 1002F pallet arrays during a stamping procedure (see Experimental Section). To demonstrate the feasibility of patterning the pallet surfaces, black ink was stamped in a variety of shapes and patterns on the surface of the pallet array (Fig. 2). In Fig. 2A, 50\% of the central region of each pallet was stamped. In contrast, in Fig. 2B only a small portion (4\%) of the center of each pallet was coated. The outer most $20-\mu \mathrm{m}$ edge of pallets were also easily inked (Fig. $2 \mathrm{C}$ ). To evaluate the precision of stamping, arrays with $100 \times 100$ elements $(130-\mu \mathrm{m}$ square pallets, 10,000 pallets) were evaluated to determine the spatial misalignment of a $30-\mu \mathrm{m}$ circle stamped at the center of each pallet. For a perfectly positioned circle, the center of the circle was $65 \mu \mathrm{m}$ from all pallet edges. Across the array, the center of the circle varied from this perfect alignment by $13 \% \pm 8 \%$ ( $n=10$ arrays). Higher precision masks and molds would further improve the centering of the circle on the pallets across the array; however, for most applications this small variation in the circle center from the pallet center was acceptable. 
To broaden the application of the stamped pallet arrays and to further demonstrate the precision and reliability of the custom-alignment tool, multi-step stamping on a single array was also performed. Each pallet could be stamped serially to place different materials onto the arrays (Figs. 2D-F). Evaluation of multiple arrays $(n=10)$ printed sequentially with a $30-\mu \mathrm{m}$ circle of black ink (Fig. 2D) demonstrated $11 \% \pm 9 \%$ variation in distance between the circle centers stamped on pallets across an array of 10,000 pallets (100 rows $\times 100$ columns). These results demonstrated the feasibility of patterning coatings in spatially discrete locations on the pallet top surfaces.

\subsection{Cell patterning on pallets in arrays}

To determine whether an extracellular matrix (ECM) could be stamped onto an array and then used to place cells at specific locations on a pallet surface $(130 \times 130 \mu \mathrm{m})$, pallet arrays were patterned with fibronectin in the center $(90 \times 90 \mu \mathrm{m}$ area $)$. H1299 cells expressing eGFP were then cultured at high density ( $>25$ cells/pallet) for 8 hours on the fibronectinpatterned pallet arrays (Figs. 3A \& B). Control arrays in which fibronection coated the entire pallet top surface were also assessed. On the center-stamped pallets, $80 \% \pm 7 \%$ of the H1299 cells ( $n=65$ pallets from 4 experiments) grew in the central stamped region. While the surface of the $1002 \mathrm{~F}$ photoresist is hydrophobic, this surface does display charged $\mathrm{O}^{-}$ groups and consequently is not highly efficient at preventing cell attachment. It may be possible to localize a greater percentage of the cells by stamping a cell repellant layer around the pallet edges as was demonstrated in Fig. 2C. For the arrays in which the entire pallet surface was covered with fibronectin, only $21 \% \pm 6 \%$ ( $\mathrm{n}=77$ pallets, 4 trials) of these cells were located in the central $90 \times 90 \mu \mathrm{m}$ region of the pallet. The paucity of cells in the center of these pallets was due to the preference of the cells for adhesion to the rough pallet edges. When cells were cultured at low density ( 5 cells/pallet) on the pallets covered entirely with fibronectin, the cells were readily seen to localize near the edges of the pallet (Fig. 3C). In contrast at low density, cells on the fibronectin-patterned pallets clearly preferred the central pallet region (Fig. 3D).

To determine whether other cell types might also be localized to the central pallet regions, MEF cells were seeded at a ratio of 1 cell to 1 pallet on arrays with fibronectin stamped in the pallet center $(90 \times 90 \mu \mathrm{m})$. The arrays were placed in culture for $12 \mathrm{~h}$. Growth of MEF cells on control arrays in which fibronection was stamped on the entire pallet top surface was also assessed. For the center-stamped arrays, on average $85 \% \pm 4 \%(\mathrm{n}=261$ pallets, 4 trials) of the MEFs were positioned with the main cell body located in the central $90 \times 90$ $\mu \mathrm{m}$ of a pallet (Fig. 4B). MEFs typically have cell diameters up to $120 \mu \mathrm{m}$ and the periphery of the cells frequently extended out beyond the patterned $90 \times 90-\mu \mathrm{m}^{2}$ area. For comparison, the main cell body of MEFs cultured on arrays without patterned fibronectin was located in the central $90 \times 90 \mu \mathrm{m}$ of a pallet only $25 \% \pm 3 \%(\mathrm{n}=409$ pallets, 4 trials) of the time (Fig. 4A). Similar results to that of H1299 and MEF cells were also obtained when HeLa cells were cultured on center-stamped pallets (Fig. S2).

\subsection{High-resolution fluorescence imaging of cells on pallet arrays}

Prior measurements of fluorescence at high spatial resolution on the pallets were hindered by intense scattering of light from the pallet edges. Indeed, the scattered light at the edge of the pallet was often nearly equal in intensity to that from the cell. To determine whether localization of the cell to the pallet center and away from the edges could spatially separate cellular fluorescence from this optical artifact, eGFP-expressing H1299 and RhoA biosensor-expressing MEF cells were cultured on fibronectin-patterned and control pallets and imaged by fluorescence microscopy. When eGFP-H1299 cells cultured on control pallets were visualized, the majority of the cells resided near the edges of the pallet and the cellular fluorescence could not be distinguished from the scattered light at the pallet edge 
adjacent to the cell (Fig. 3E). In contrast, most eGFP-H1299 cells cultured on fibronectinpatterned pallets were centered on the pallet and the cellular fluorescence was well separated from the scattered light at the pallet edges (Fig. 3F).

Standard pallet arrays could not be utilized for biosensor measurements due to an array thickness that exceeded the $200 \mu \mathrm{m}$-working distance of the required high NA objectives. For measurements requiring high spatial resolution, the array was fabricated on a thin glass coverslip (150 $\mu \mathrm{m}$ thickness) with pallets $25 \mu \mathrm{m}$ in height yielding a maximal array thickness of $175 \mu \mathrm{m}$. Similar patterning quality to that of the thicker pallets was observed when these arrays were center-stamped with ECM. MEF cells expressing the RhoA biosensor were cultured on these fibronectin center-stamped and control pallet arrays. RhoA activation was measured as the ratio of FRET (the fluorescence of the acceptor fluorophore YFP upon CFP excitation) divided by the fluorescence of CFP (the donor fluorophore). The preferred attachment of the MEFs near the pallet edges on the control arrays prevented measurement of the RhoA sensor properties in the cell. Fluorescence images of both FRETbased YFP emission and CFP emission displayed intense scatter at the pallet borders (Fig. 4C). This was particularly problematic since RhoA is most active at the cell edges [6, 7]. When cells were cultured on the center-stamped arrays, the majority of the cell including the cell borders were spatially separated from the pallet edges. Cellular FRET-based YFP emission and CFP emission were also spatially segregated from the pallet-edge light scatter (Fig. 4D). The cells displayed RhoA activation at the edge of cellular protrusions ( $\mathrm{n}=7$ cells) consistent with prior measurements (Fig. 5) [6]. In addition changes in RhoA activation were easily observed during constitutive protrusion/retraction cycles for cells on the center-stamped pallets.

\section{Conclusion}

This work demonstrates the successful surface patterning of three-dimensional microstructures using a stamp aligned with a custom tool fitted onto a microscope. Most patterning for cell culture is performed on a two-dimensional surface so that no alignment with the surface is required [17-19]. In contrast surface patterning of a microstructure requires precise placement of the molecules to be patterned with respect to the microstructure itself. Common strategies to accomplish this goal include the use of photolithography and microfluidic channels as well as tailored dewetting of surfaces [20-23]. These methods can be complex requiring expensive equipment, additional microfabricated devices (microchips with channels), or a strategy to tailor surface tension. The current method uses an easily fabricated alignment piece mounted onto a microscope for micron-resolution alignment and patterning and thus may be of general utility in microdevice construction.

A goal of this work was to demonstrate the feasibility of performing highly sensitive and spatially resolved fluorescence measurements on the surface of the pallet arrays. This achievement has the potential to enable cell selection and separation based on dynamic spatial phenomena which would greatly broaden the type of selection criteria used to separate a mixed population of cells. While the image scanning and analysis demonstrated with the pallet technology in the current application were low in throughput, clear avenues exist to enhance and speed image acquisition and analysis utilizing methods from image cytometry. Future work for this technology will focus on the integration of automated image processing followed by identification and isolation of cells with novel signaling properties.

\section{Supplementary Material}

Refer to Web version on PubMed Central for supplementary material. 


\section{Acknowledgments}

This research was supported by the National Institutes of Health (EB007612, GM057464). We also acknowledge the financial support provided by the Carolina Center for Cancer Nanotechnology Excellence (CCNE) and the American Cancer Society/The Josephine Peiser Charitable Foundation Postdoctoral Fellowship \#PF-07-102-01TBE (J.S.Z.). Professor Adrienne Cox and Dr. Jared Snider are greatly acknowledged for kindly providing the eGFP H1299 cell lines.

\section{References}

1. Ghosh M, Ichetovkin I, Song X, Condeelis JS, Lawrence DS. J. Am. Chem. Soc. 2002; 124:2440. [PubMed: 11890784]

2. Levskaya A, Weiner OD, Lim WA, Voigt CA. Nature. 2009; 461:997. [PubMed: 19749742]

3. Wu YI, Frey D, Lungu OI, Jaehrig A, Schlichting I, Kuhlman B, Hahn KM. Nature. 2009; 461:104. [PubMed: 19693014]

4. Zhang J, Allen MD. Molecular BioSystems. 2007; 3:759. [PubMed: 17940658]

5. Kraynov VS, Chamberlain C, Bokoch GM, Schwartz MA, Slabaugh S, Hahn KM. Science. 2000; 290:333. [PubMed: 11030651]

6. Pertz O, Hodgson L, Klemke RL, Hahn KM. Nature. 2006; 440:1069. [PubMed: 16547516]

7. Machacek M, Hodgson L, W. C. Elliott H, Pertz O, Nalbant P, Abell A, Johnson GL, Hahn KM, Danuser G. Nature. 2009; 461:99. [PubMed: 19693013]

8. Salazar, G.T.a.; Wang, Y.; Young, G.; Bachman, M.; Sims, CE.; Li, GP.; Allbritton, NL. Anal. Chem. 2007; 79:682. [PubMed: 17222037]

9. Wang Y, Young G, Bachman M, Sims CE, Li GP, Allbritton NL. Anal. Chem. 2007; 79:2359. [PubMed: 17288466]

10. Shadpour H, Sims CA, Allbritton NL. Cytometry A. 2009; 75:609. [PubMed: 19504569]

11. Shadpour H, Sims CA, Thresher RJ, Allbritton NL. Cytometry A. 2009; 75:121. [PubMed: 19012319]

12. Wang Y, Young G, Aoto Phillip C, Pai J-H, Bachman M, Li GP, Sims CE, Allbritton NL. Cytometry A. 2007; 71:866. [PubMed: 17559133]

13. Shadpour H, Allbritton NL. ACS Appl. Mater. Interfaces. 2010; 2:1086. [PubMed: 20423129]

14. Pai J-H, Wang Y, Salazar GTA, Sims CE, Bachman M, Li GP, Allbritton NL. Anal. Chem. 2007; 79:8774. [PubMed: 17949059]

15. Morgenstern JP, Land H. Nucleic Acids Res. 1990; 18:3587. [PubMed: 2194165]

16. Hodgson, L.; Shen, F.; Hahn, KM. Curr. Protoc. Cell Biol. Bonifacino, JS.; Dasso, M.; Harford, JB.; Lippincott-Schwartz, J.; Yamada, KM., editors. John Wiley \& Sons, Inc.; Hoboken, NJ: 2010. p. 1

17. El-Ali J, Sorger PK, Jensen KF. Nature. 2006; 442:403. [PubMed: 16871208]

18. Ito Y. Biomaterials. 1999; 20:2333. [PubMed: 10614939]

19. Roth EA, Xu T, Das M, Gregory C, Hickman JJ, Boland T. Biomaterials. 2004; 25:3707. [PubMed: 15020146]

20. Hou HJ, Kim W, Grunlan M, Han A. J. Micromech. Microeng. 2009; 19

21. Li W, Nie Y, Zhang JH, Zhu DF, Li X, Sun HZ, Yu K, Yang B. Macromol. Chem. Phys. 2008; 209:247.

22. Revzin A, Russell RJ, Yadavalli VK, Koh WG, Deister C, Hile DD, Mellott MB, Pishko MV. Langmuir. 2001; 17:5440. [PubMed: 12448421]

23. Yan J, Pedrosa VA, Simonian AL, Revzin A. ACS Appl. Mater. Interfaces. 2010; 2:748. [PubMed: 20356276] 


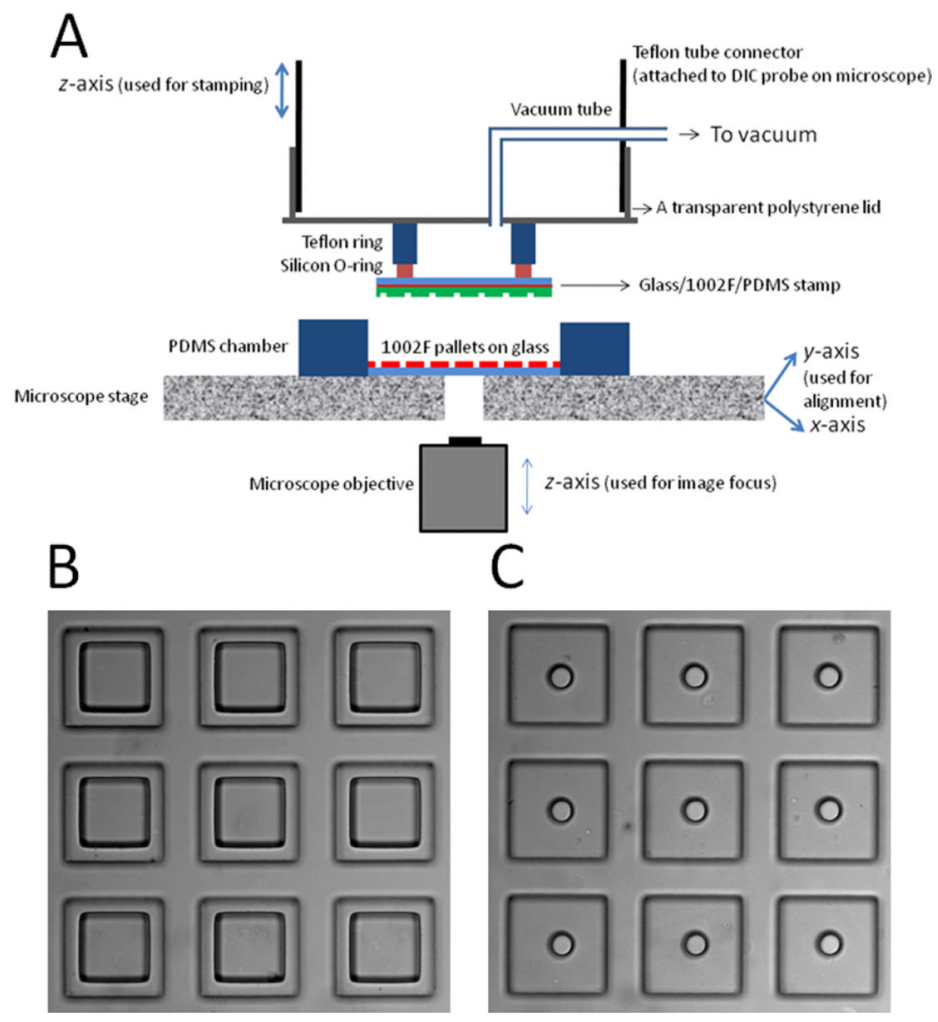

Fig. 1.

Printing an array with ink. A) Schematic of the customized alignment tool for stamping of the pallet arrays. B \& C) Micrographs of square $(90 \times 90 \mu \mathrm{m})$ and circular $(30-\mu \mathrm{m}$ diameter $)$ patterns on PDMS stamps aligned over a pallet array possessing $130-\mu \mathrm{m}$ square pallets. 

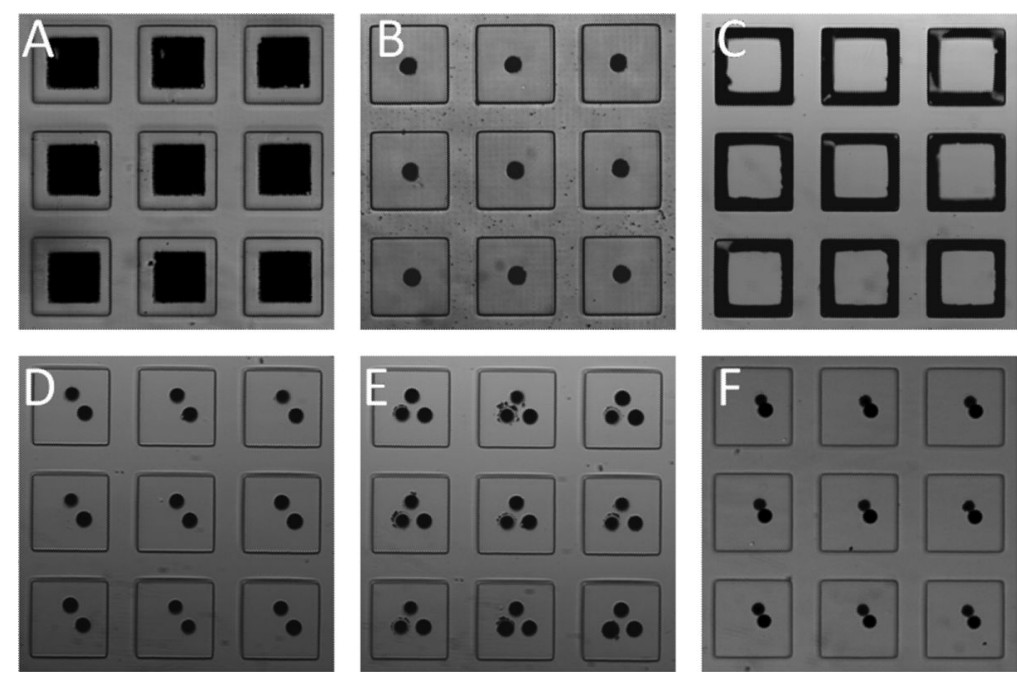

Fig. 2.

Micrographs of black ink stamped on 130- $\mu \mathrm{m}$ square pallets. A \& B) A 90- $\mu \mathrm{m}$ square (A) or $30-\mu \mathrm{m}$ circle (B) stamped at the center of each pallet. C) A 20- $\mu \mathrm{m}$ wide frame printed around the edges of each pallet. D, E) Sequential stamping of two or three 30- $\mu \mathrm{m}$ circles with the same ink. (F) Sequential stamping of two different color inks. The smaller circle (upper left) was a red ink which exhibited differential spreading relative to the black ink (lower right circle). 

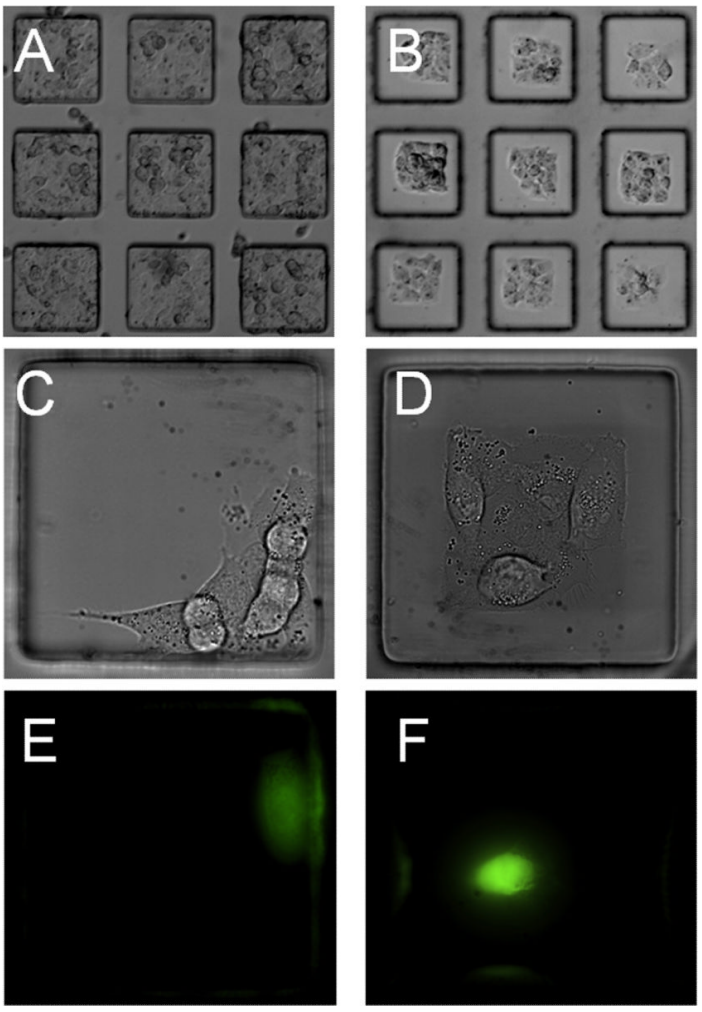

Fig. 3.

Images of eGFP-expressing H1299 cells. DIC (A-D) and fluorescence (E-F) micrographs of H1299 cells plated on $1002 \mathrm{~F}$ pallets $(130 \times 130 \mu \mathrm{m})$ that were coated with fibronectin. A \& C) Pallets in which the entire top surface was stamped with fibronectin. B \& D) Pallets in which a $90 \times 90 \mu \mathrm{m}$ squared centered on the pallet surface was stamped with fibronectin. $\mathrm{E}$ $\&$ F) Fluorescence micrographs $(40 X$, oil objective with NA $=1.30)$ of control $(E)$ and fibronectin center-stamped (F) pallets with an H1299 cell. 

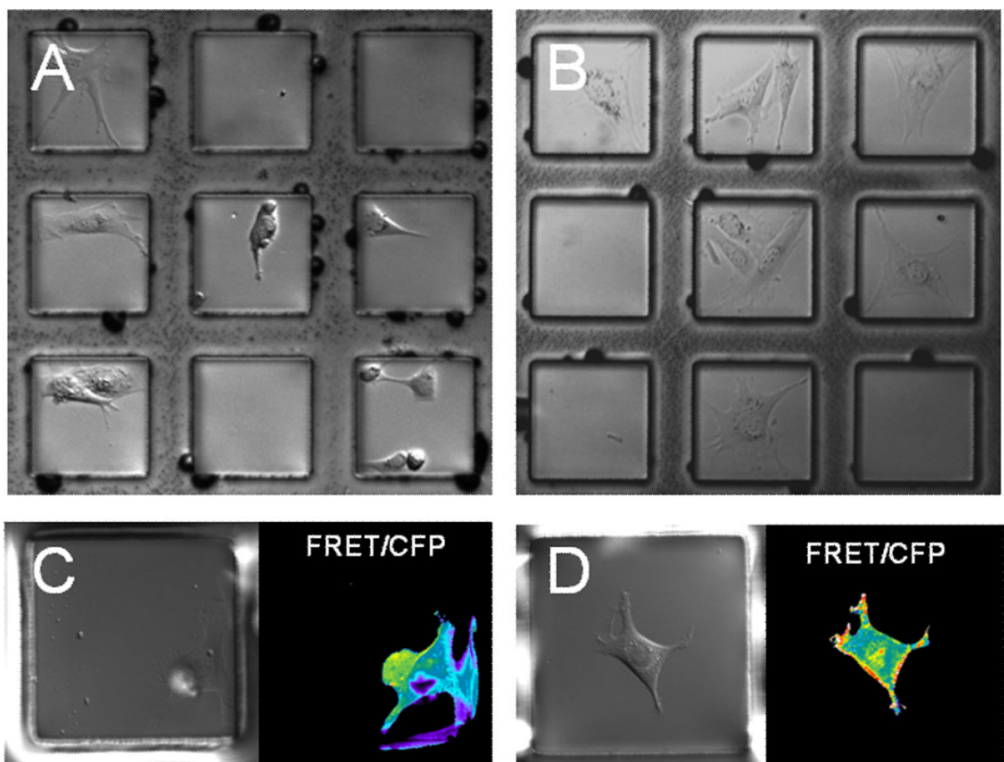

\section{FRET/CFP}
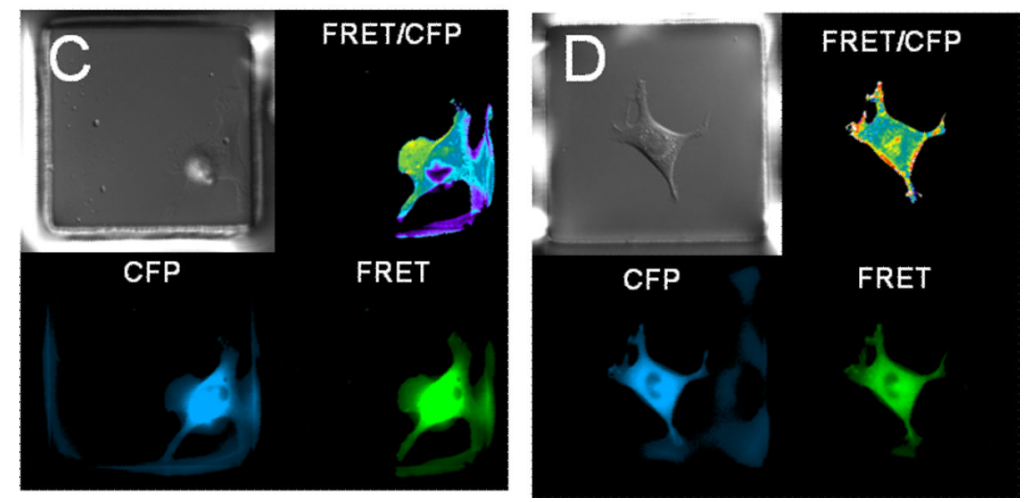

Fig. 4.

Images of MEF cells expressing the fluorescent RhoA biosensor. A \& B) DIC micrographs of MEF cells cultured on $1002 \mathrm{~F}$ pallet arrays that were stamped with fibronectin on their entire top surface (A) and on the central $90 \times 90 \mu \mathrm{m}$ region (B). C \& D) Images of RhoA biosensor in MEF cells on a control array (C) and a center-stamped array (D). All pallet arrays were $130 \mu \mathrm{m}$ square. The FRET-YFP/CFP images are pseudo-colored images with warmer colors representing the greatest RhoA activity (highest FRET /CFP ratio) 


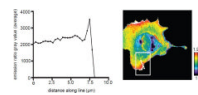

Fig. 5.

RhoA activity in the leading edge of a cell on a center-stamped pallet. Right panel: Shown is the FRET YFP/CFP image of a cell expressing the RhoA biosensor. The white box highlights the region of the cell used for analysis. The dashed line represents the location of the line scan shown in the left panel. The color bar defines the dynamic range of the FRET YFP/CFP ratio. Left panel: Shown is a graph of the FRET YFP/CFP ratio (y axis) vs. distance (x axis). The distance numerically increases towards the outer edge of the cell. 